\title{
Dual-Wavelength Continuous Wave Photoacoustic Doppler Flow Measurement
}

\author{
Hongcai ZHAO ${ }^{1,3}$, Yanbin $\mathrm{LIU}^{2}$, Tayyab FAROOQ ${ }^{2}$, and Hui FANG ${ }^{2 *}$ \\ ${ }^{1}$ Institute of Modern Optics, Nankai University, Tianjin 300350, China \\ ${ }^{2}$ Institute of Microscale Optoelectronics, Nanophotonics Research Center \& Shenzhen Key Laboratory of Micro-Scale \\ Optical Information Technology, Shenzhen University, Shenzhen 518060, China \\ ${ }^{3}$ HMN Technologies Co., Ltd., Tianjin 300467, China \\ *Corresponding author: Hui FANG_E-mail: fhui79@szu.edu.cn
}

\begin{abstract}
Photoacoustic Doppler flow measurement based on continuous wave laser excitation owns the merit of clearly presenting the Doppler power spectra. Extending this technique to dual wavelengths can gain the spectral information of the flow sample extra to the flow speed information. An experimental system with two laser diodes respectively operated at $405 \mathrm{~nm}$ and $660 \mathrm{~nm}$ wavelengths is built and the flow measurement with black and red dyed polystyrene beads is performed. The measured Doppler power spectra can vividly reflect the flow speed, the flow direction, as well as the bead color. Since it is straightforward to further apply the same principle to multiple wavelengths, we can expect this type of spectroscopic photoacoustic Doppler flow measurement will be developed in the near future which will be very useful for studying the metabolism of the slowly moving red blood cell inside microvessels.
\end{abstract}

Keywords: Photoacoustic Doppler; Doppler power spectra; multispectral photoacoustics; flow measurement; continuous wave laser

Citation: Hongcai ZHAO, Yanbin LIU, Tayyab FAROOQ, and Hui FANG, "Dual-wavelength Continuous Wave Photoacoustic Doppler Flow Measurement," Photonic Sensors, 2022, 12(1): 1-9.

\section{Introduction}

After nearly three decades of continuous endeavor, the photoacoustic imaging has matured from a physical phenomena interrogation technology to real clinical applications [1-3]. The photoacoustic signal generation involves the energy transformation process started from light absorption to ultrasound generation, and it is common to use the Q-switched pulse laser as the excitation light source and construct the image based on the time domain signal processing. However, in a few years recently, the frequency domain photoacoustic imaging based on discrete sinuous modulated waves has attracted renewed interests after its demonstration on imaging blood vessels a decade ago [4]. The major impetus for this development is that when considering the multispectral photoacoustic imaging, diode lasers are available on average every $20 \mathrm{~nm}$ in the range from the near ultraviolet to the near infrared while they are much less expensive than an optical parametric oscillator (OPO) or a dye laser [5]. Although this continuous wave based modality seems suffer from the poor depth discrimination and low signal to noise ratio, in some cases such as for imaging thin slice or cultured cells the depth

Received: 10 July 2020 / Revised: 25 February 2021

(C) The Author(s) 2021. This article is published with open access at Springerlink.com

DOI: $10.1007 / \mathrm{s} 13320-021-0633-6$

Article type: Regular 
information is usually irrelevant, and the low signal to noise ratio can be compensated well by the narrowband detector such as a lock-in detector $[5,6]$. Beside the advantage of operating simultaneously at several discrete wavelengths [7], the modulation frequency can be easily tuned and the multi-frequency amplitude along with phase can provide valuable information for constructing structures at different scales $[8,9]$. Moreover, when working in frequency sweeping modality, the depth information can be accessed $[10,11]$.

The importance of the continuous wave photoacoustic measurement has also been recognized in the recent work of quantizing the blood flow based on analyzing the photoacoustic Doppler shift [9]. The photoacoustic Doppler effect was demonstrated previously [12-14], and since then the photoacoustic Doppler flow measurement with depth discrimination was also realized with the tone burst excitation $[15,16]$. As recently pointed out by us [17], although there are already several technologies based on pulsed laser excitation also coined with photoacoustic Doppler, the unique feature of the continuous wave photoacoustic Doppler measurement is the clear presentation of the Doppler power spectra, from which not only the flow speed, but also the flow angle and the Doppler broadening can be extracted. Among various developing photoacoustic flow measurements [18], the continuous wave method presents an affordable, fast, and accurate alternative when the information of the flow depth is not critical. Here, we make a further step forward: by combing two continuous wave laser beams with different wavelengths and modulating the intensity of the two laser beams at different modulation frequencies, we demonstrate the dual-wavelength photoacoustic Doppler measurement. We show that the dual-wavelength Doppler power spectra contain the spectral information of flowing beads with different colors. Since extending this method to multiple wavelengths is straightforward, the technique of spectroscopic photoacoustic Doppler flow measurement based on the same principle can be well expected.

\section{Materials and method}

\subsection{Experimental system}

The layout of our experimental system is illustrated in Fig. 1. The light source is composed of two diode laser devices which operate respectively with a laser diode with the red wavelength $(660 \mathrm{~nm}$, $120 \mathrm{~mW}$, HL6545MG, Thorlabs) and a laser diode with the blue wavelength (405 nm, $120 \mathrm{~mW}$, ML320G2-11, Thorlabs). Each of the diode laser devices includes a thermo-electrically cooled mounts (TCLDM9, Thorlabs) and a temperature controller (ITC4005, Thorlabs). The diode laser beams are respectively coupled with the "Lens 1" and "Lens 2" (C230220P-B, Thorlabs) into a wavelength division multiplexer (with optical fibers of $N A=0.22$ and core diameter of $105 \mu \mathrm{m}$, Nanjin Chunhui). Finally, the laser output from the multiplexer optical fiber is collimated with an achromatic objective (UPLANFLN 10x ,Olympus) and focused onto the flow sample by the "Lens 3" (with the focal length $3.5 \mathrm{~mm}$, AC254-035-A-ML, Thorlabs). The photoacoustic waves generated by the flow sample is detected by a focused water-immersion ultrasound transducer (with the central operation frequency $2.25 \mathrm{MHz}$, bandwidth $0.71 \mathrm{MHz}$, diameter $0.5 \mathrm{inch}$, focal length 0.8 inch, i4-0208-P-SU-F0.80IN-PTF, Harisonic). The photoacoustic signal is further amplified through a preamplifier (5660C, Olympus) and analyzed by a lock-in amplifier (7280, Signal Recovery). A microfluidic pump (LSP01-2A, Longer) is used for controlling the flow speed of the flow sample.

As depicted in Fig. 1, both diode lasers are separately modulated with a waveform generator (AFG3102, Tektronix) and these two waveform generators are synchronized by connecting their $10 \mathrm{MHz}$ clocks. The modulation frequencies for the two diode lasers are set differently which are 
$f_{0}+\Delta f_{1}=1.9 \mathrm{MHz}+5 \mathrm{~Hz}$ for the blue diode laser and $f_{0}+\Delta f_{2}=1.9 \mathrm{MHz}+15 \mathrm{~Hz}$ for the red diode laser, respectively. Here, the frequency $f_{0}=1.9 \mathrm{MHz}$ works as the reference frequency and is set with the reference signal fed into the lock-in amplifier. As a result, the demodulated Doppler power spectra will show two similar profiles around $5 \mathrm{~Hz}$ and $15 \mathrm{~Hz}$.

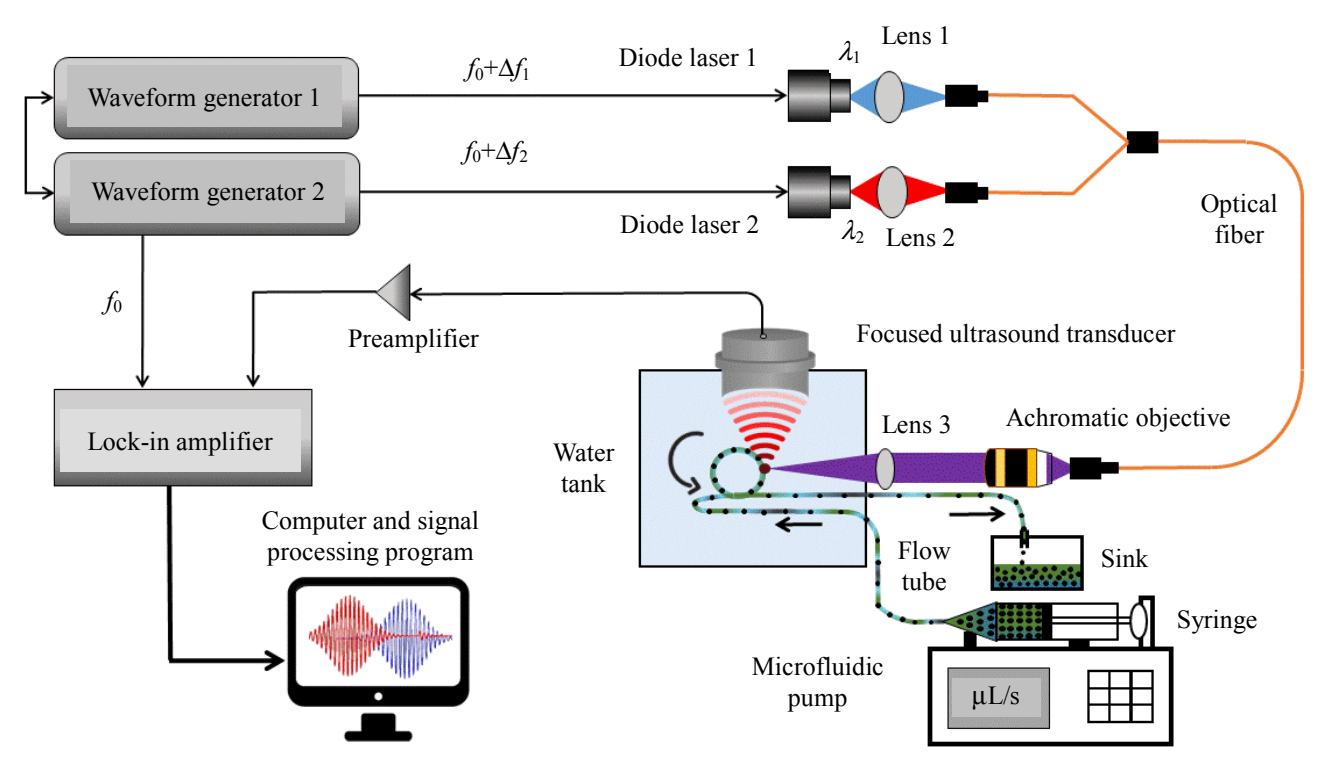

Fig. 1 Schematic of the experimental system of the dual-wavelength photoacoustic Doppler flow measurement.

\subsection{Materials}

As illustrated in Fig. 1, the flow is sustained inside a transparent tygon tube (Saint-Gobain) with an inner diameter of $0.5 \mathrm{~mm}$ and an outer diameter of $1.5 \mathrm{~mm}$. The flow sample is created by suspending either black dyed beads or red dyed beads in distill water with the final volume concentration of $5 \%$. The dyed beads (dyed polystyrene beads, Duke Scientific) have the mean diameter of $50 \mu \mathrm{m}$ and their mass density is about $1.05 \mathrm{~g} / \mathrm{cm}^{3}$. An appropriate amount of sodium metatungstate (Sigmal-Aldrich) is solved into the suspension to match the mass density so that the beads can stay uniformly in the solution for a few hours. Also, several drops of Tween-20 reagent (Sigmal-Aldrich) are added and mixed to prevent the bead aggregation.

\subsection{Signal processing method}

As discussed previously [17], when the laser modulation frequency is set as $f_{0}+\Delta f$ with an offset $\Delta f$ away from the reference frequency $f_{0}$, the upward or downward flow direction can be identified by observing the peak position of the photoacoustic Doppler power spectrum relative to the demodulated frequency $\Delta f$. There is an essential step that the demodulated two-channel output $x(t)$ and $y(t)$ from the lock-in amplifier should be combined and processed as

$$
\left\{\begin{array}{l}
s(t)=x(t)+\mathrm{i} y(t) \\
b(t)=x(t)-\mathrm{i} y(t)
\end{array}\right.
$$

such that when power spectra of $s(t)$ and $b(t)$ are both plotted, the signal to noise ratio can be clearly discerned [Here $s(t)$ is defined as the photoacoustic signal while $b(t)$ as the background signal].

As shown in Fig. 2 for the results of the horizontal flow and in Fig. 3 for the results of the vertical flow, the experimental power spectrum is 
first smoothed and then fitted with a curve including two Gaussian profiles near $\Delta f_{1}=5 \mathrm{~Hz}$ and $\Delta f_{2}=15 \mathrm{~Hz}$ in order to obtain the two peak positions, the two widths, and the two amplitudes. The identical shape is assumed for the two separated distribution profiles since they correspond to the same flow [the expression can be found in (2) and (3)]. The flow speed then can be extracted from the fitted width for the horizontal flow or from the fitted

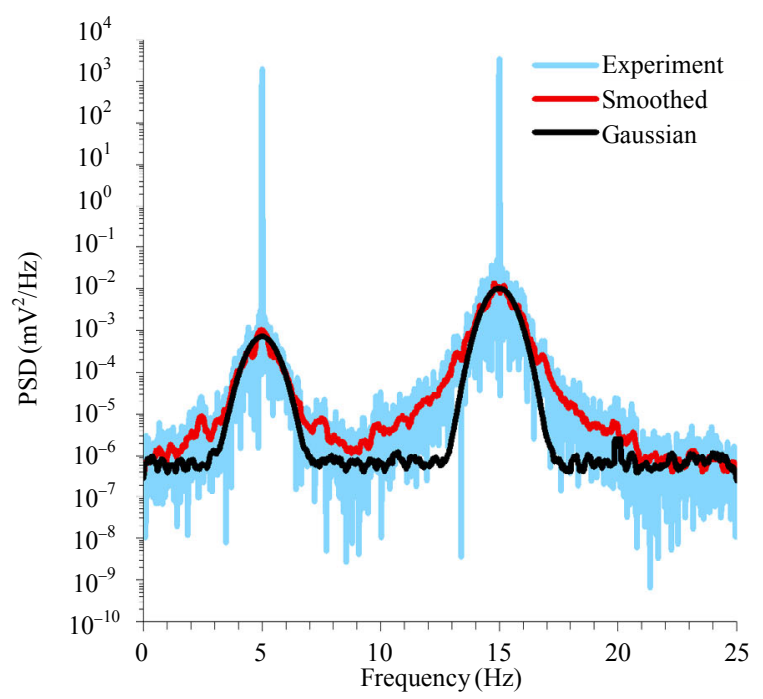

(a)

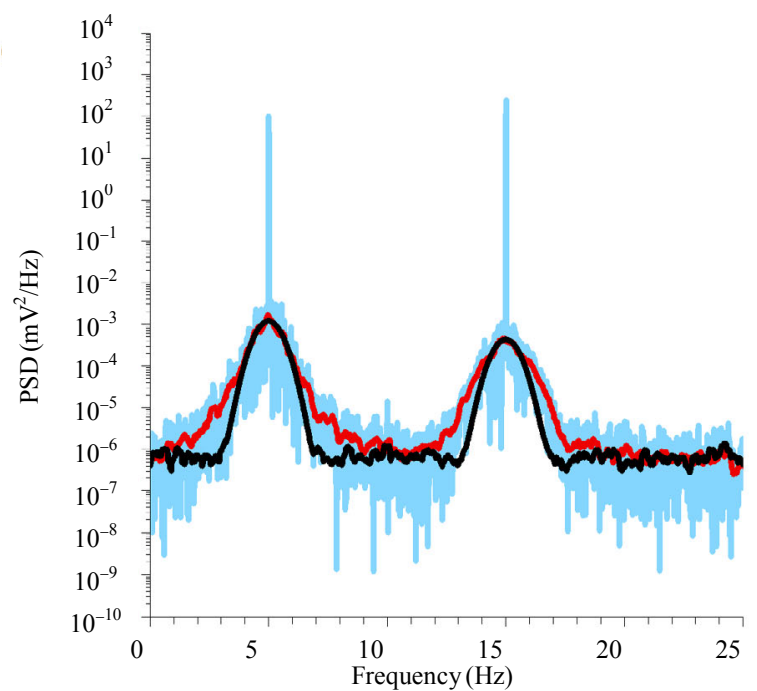

(c) peak position shift relative to $5 \mathrm{~Hz}$ and $15 \mathrm{~Hz}$ for the vertical flow. In the current study with the same color of beads for each flow sample, the accuracy of measuring the flow speed has not been improved compared with the single wavelength measurement. However, the advantage is that the spectral information of the beads can be extracted by analyzing the ratio between the fitted two amplitudes.

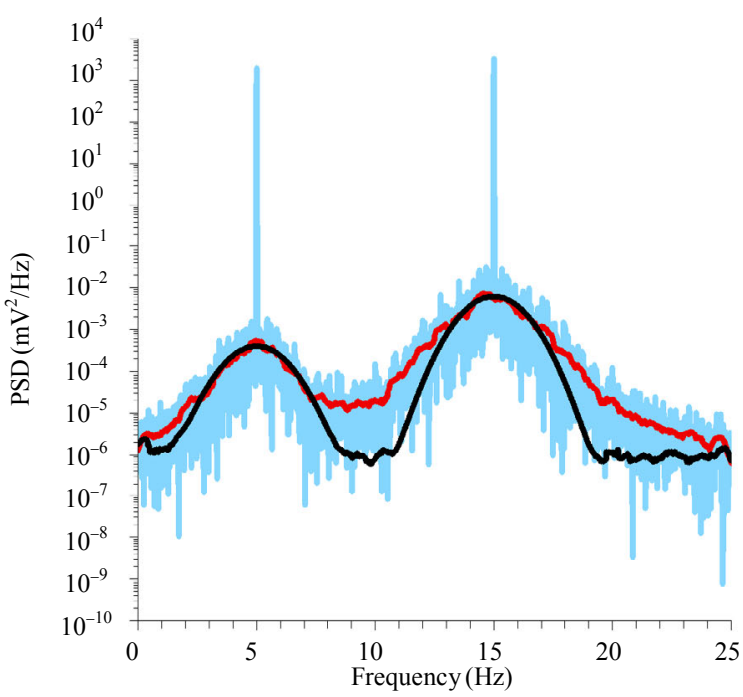

(b)

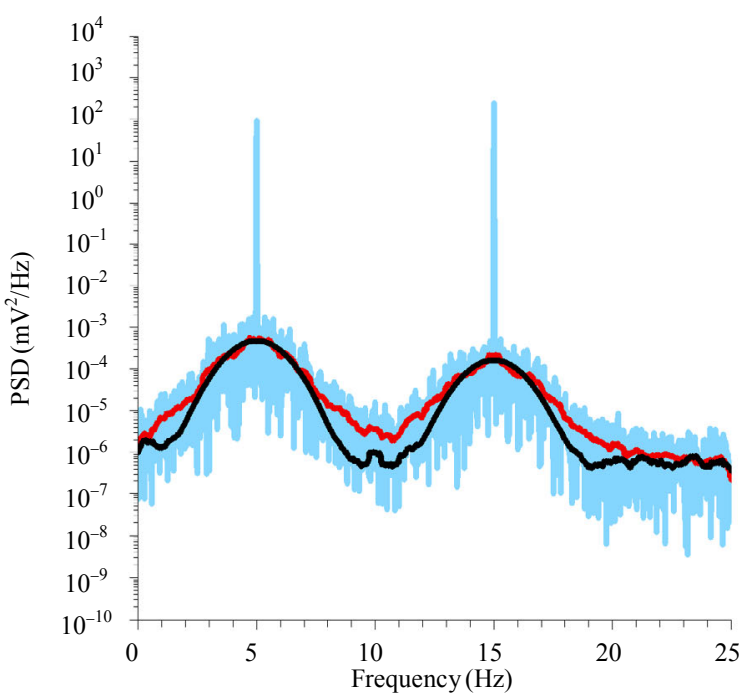

(d)

Fig. 2 Photoacoustic Doppler power spectra plotted in log scale for horizontal flow: (a) and (b) for the flows of the black dyed bead sample with the average flow speed settings of $0.2 \mathrm{~mm} / \mathrm{s}$ and $0.4 \mathrm{~mm} / \mathrm{s} ;(\mathrm{c})$ and (d) for the flows of the red dyed bead sample with the average flow speed settings of $0.2 \mathrm{~mm} / \mathrm{s}$ and $0.4 \mathrm{~mm} / \mathrm{s}$. 


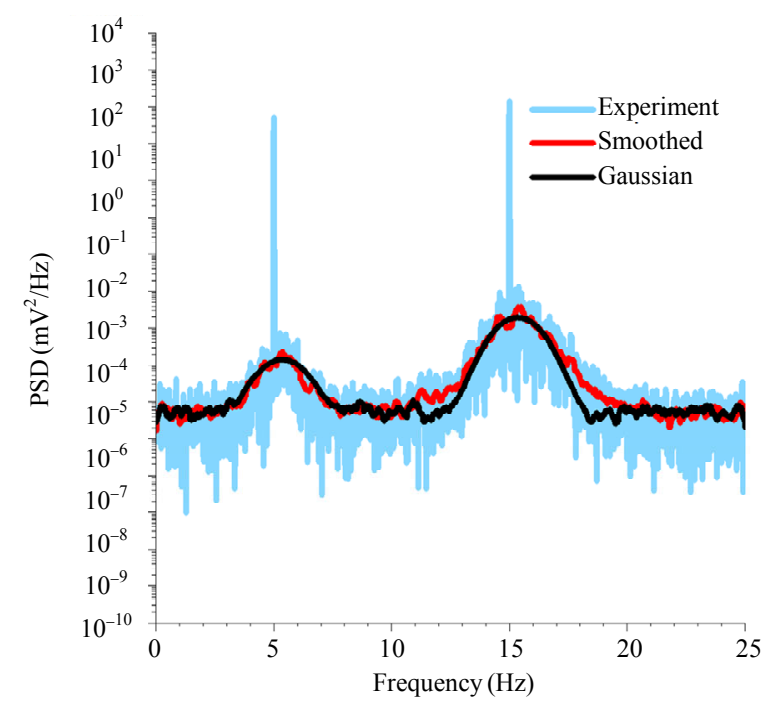

(a)

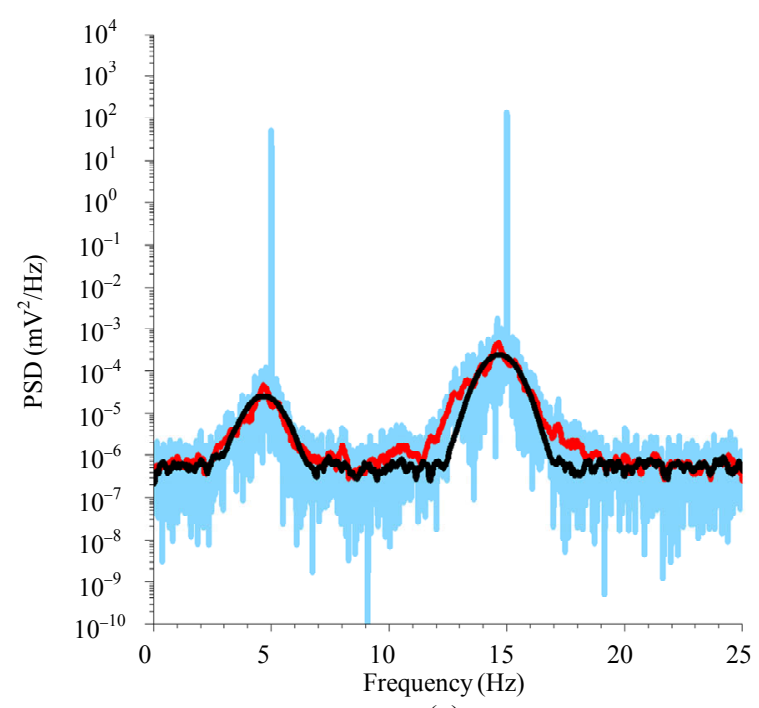

(c)

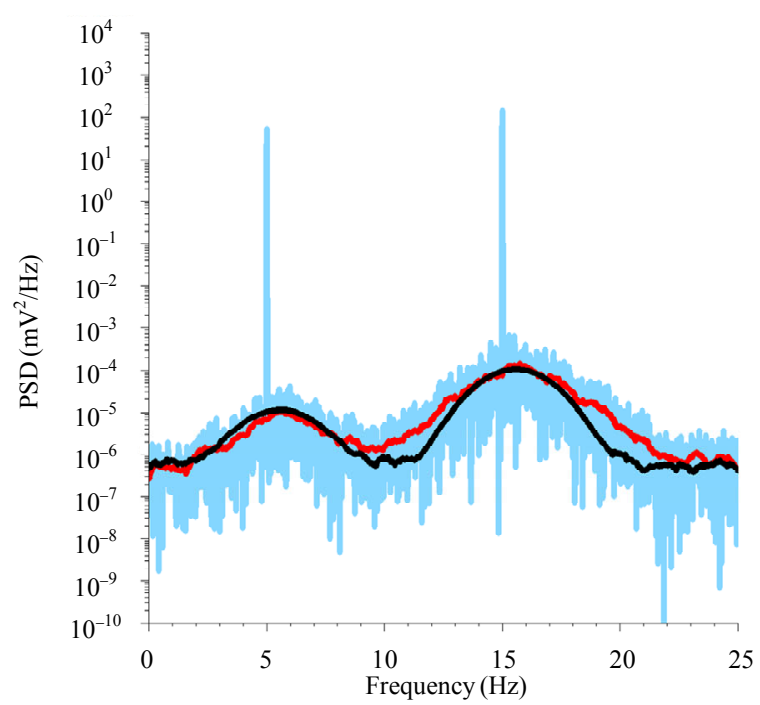

(b)

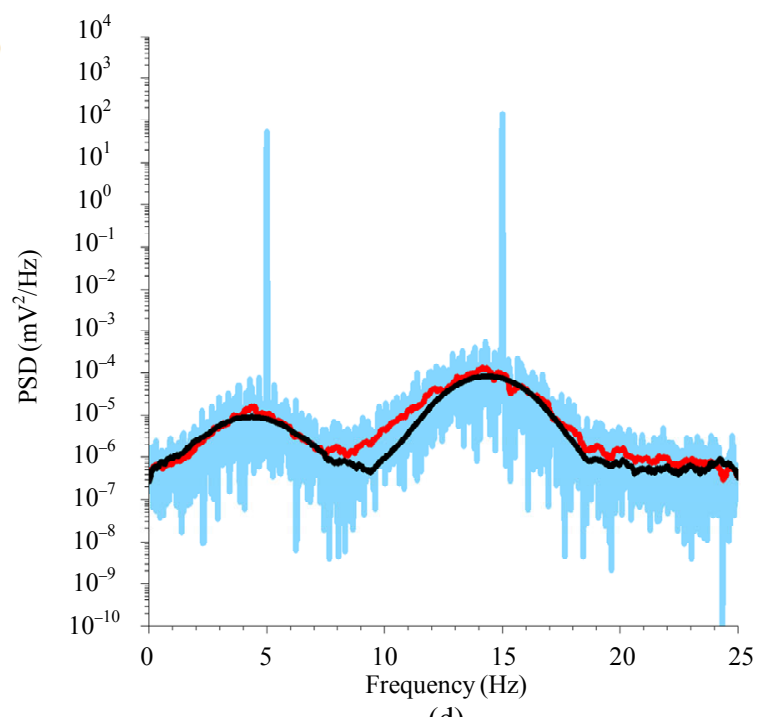

(d)

Fig. 3 Photoacoustic Doppler power spectra plotted in log scale for vertical flow measured for the black dyed bead sample: (a) and (b) for the upward flows towards to the ultrasound transducer with the average flow speed settings of $0.2 \mathrm{~mm} / \mathrm{s}$ and $0.4 \mathrm{~mm} / \mathrm{s}$; (c) and (d) for the downwards flows away from the ultrasound transducer with the average flow speed settings of $0.2 \mathrm{~mm} / \mathrm{s}$ and $0.4 \mathrm{~mm} / \mathrm{s}$.

\section{Results}

Here, we present the photoacoustic Doppler power spectra measured for the average flow speed settings of $0.2 \mathrm{~mm} / \mathrm{s}$ and $0.4 \mathrm{~mm} / \mathrm{s}$. Both flow samples prepared either from the black dyed beads or from the red dyed beads were measured in the flow angles of $90^{\circ}$ and $0^{\circ}$ (respectively termed as horizontal flow and vertical flow). However, for the vertical flow measurement, the photoacoustic signal of red dyed beads excited by the red diode laser is too weak. Therefore, for this flow angle, only the results of measuring black dyed beads are presented. To obtain the smoothed curves as shown in Figs. 2 and 3 , the moving average window with the respective width of $0.26 \mathrm{~Hz}$ or $0.51 \mathrm{~Hz}$ (corresponding to 26 or 51 data points) is applied for the measured signals related to $0.2 \mathrm{~mm} / \mathrm{s}$ or $0.4 \mathrm{~mm} / \mathrm{s}$ flow.

\subsection{Horizontal flow results}

For the horizontal flow (with the flow angle of $90^{\circ}$, referring to Fig. 1), we measure the top portion of the circle formed by the flow tube and plotted the 
results in Fig. 2. Figures 2(a) and 2(c) show the photoacoustic Doppler power spectra for the flows with average flow speed settings of $0.2 \mathrm{~mm} / \mathrm{s}$, Figs. 2(b) and 2(d) show those for the flow of $0.4 \mathrm{~mm} / \mathrm{s}$. At the same time, Figs. 2(a) and 2(b), are both for the black dyed bead sample, while Figs. 2(c) and 2(d) are both for the red dyed bead sample.

In Fig. 2, the light blue curves are the experimental photoacoustic Doppler spectra, the red curves are the smoothed results, and the black curves are the best Gaussian fits with the formula of

$$
\begin{array}{r}
g\left(a_{1}, a_{2}, \sigma, f\right)=a_{1} \exp \left[-(f-5)^{2} /\left(2 \sigma^{2}\right)\right]+ \\
a_{2} \exp \left[-(f-15)^{2} /\left(2 \sigma^{2}\right)\right]+b(f)
\end{array}
$$

where $b(f)$ represents the power spectra of the background signal discussed in (1), $a_{1}$ and $a_{2}$ are the fitted Gaussian amplitudes, and $\sigma$ is the fitted Gaussian width. This formula can be considered as a good approximation because the photoacoustic Doppler power spectrum due to the blue (or the red) diode laser excitation should be centered at $5 \mathrm{~Hz}$ (or $15 \mathrm{~Hz}$ ) respectively and should both be symmetric and with the same width. In Table 1, the fitted parameters according to (2) for all of the four Gaussian fits are listed.

Table 1 Fitted parameters by (2) for all of the Gaussian fits shown in Fig. 2.

\begin{tabular}{lccc}
\hline & $a_{1}\left(\mathrm{mV}^{2} / \mathrm{Hz}\right)$ & $a_{2}\left(\mathrm{mV}^{2} / \mathrm{Hz}\right)$ & $\sigma(\mathrm{Hz})$ \\
\hline (a) $0.2 \mathrm{~mm} / \mathrm{s}$ flow of black beads & 0.00071 & 0.01000 & 0.48 \\
(b) $0.4 \mathrm{~mm} / \mathrm{s}$ flow of black beads & 0.00040 & 0.00620 & 0.97 \\
(c) $0.2 \mathrm{~mm} / \mathrm{s}$ flow of red beads & 0.00120 & 0.00043 & 0.47 \\
(d) $0.4 \mathrm{~mm} / \mathrm{s}$ flow of red beads & 0.00047 & 0.00016 & 0.98 \\
\hline
\end{tabular}

Looking at the results shown in Fig. 2 and Table 1, first, let us compare the difference between different flow speeds, i.e., compare the figures from the left side to the right side in Fig. 2. As can be seen, for the width of the Doppler power spectral profiles, Figs. 2(a) and 2(c) show the similarity and Figs.2(b) and 2(d) show the similarity, while those in the latter two figures are clearly wider. Correspondingly very well, the fitted results of $\sigma$ shown in Table 1 accurately reflect the fact that the Doppler power spectra widths for the flow of $0.4 \mathrm{~mm} / \mathrm{s}$ should be two times of those for the flow of $0.2 \mathrm{~mm} / \mathrm{s}$. It is also interesting to observe that accompanying the doubling of the widths, the amplitudes $a_{1}$ and $a_{2}$ are both approximated decreased by half, which is true for either black dyed bead flow or red dyed bead flow. This agrees with the fact that the total photoacoustic energy should be conserved for the same sample with different flow speeds under the condition that the measuring duration is actually the same (100s).

Next, let us analyze the spectral information contained in the fitted $a_{1}$ and $a_{2}$, and compare the results in Fig. 2 from the top to the bottom. Apparently as can be seen, in both of Figs.2(a) and 2(b), the values of $a_{2}$ are higher than $a_{1}$, while in both of Figs. 2(c) and 2(d), the situation is opposite. The corresponding results in Table 1 show that the ratio $a_{2} / a_{1}$ is 14.3 and 15.5 respectively for the black dyed bead flow shown in the top two figures [Figs. 2(a) and 2(b)], and the ratio is 0.36 and 0.34 respectively for the red dyed bead flow shown in the bottom two figures [Figs.2(c) and 2(d)]. Therefore, we can unambiguously distinguish the color of the flowing dyed beads by analyzing the ratio $a_{2} / a_{1}$ of the power spectra. For the red dyed beads, the ratio $a_{2} / a_{1}$ clearly should be smaller than 1 since the red color appearance comes from the less absorption in the red waveband than that in the blue waveband.

\subsection{Vertical flow results}

As shown explicitly in Fig. 1, we can move the flow sample and let the laser focus spot to illuminate the side portion of the circled flow tube and thus study the vertical flow (with the flow angle of $0^{\circ}$ ). The experimental results along with the smoothed curves and Gaussian fitting curves are plotted in Fig. 3 , while the fitted parameters are listed in Table 2 . This time the following Gaussian fitting function is applied: 


$$
\begin{array}{r}
g\left(a_{1}, a_{2}, \sigma, f\right)=a_{1} \exp \left[-(f-5-\delta)^{2} /\left(2 \sigma^{2}\right)\right]+ \\
a_{2} \exp \left[-(f-15-\delta)^{2} /\left(2 \sigma^{2}\right)\right]+b(f)
\end{array}
$$

where the extra term not appeared in (2) is $\delta$, the shift of the Doppler power spectral peak.

Table 2 Fitted parameters by (3) for the results of Fig. 3.

\begin{tabular}{lcccc}
\hline & $a_{1}\left(\mathrm{mV}^{2} / \mathrm{Hz}\right)$ & $a_{2}\left(\mathrm{mV}^{2} / \mathrm{Hz}\right)$ & $\delta(\mathrm{Hz})$ & $\sigma(\mathrm{Hz})$ \\
\hline $\begin{array}{l}\text { (a) } 0.2 \mathrm{~mm} / \mathrm{s} \\
\text { flow }\end{array}$ & 0.000013 & 0.00019 & 0.35 & 0.75 \\
$\begin{array}{c}\text { (b) } 0.4 \mathrm{~mm} / \mathrm{s} \text { upward } \\
\text { flow }\end{array}$ & 0.000011 & 0.00010 & 0.57 & 1.25 \\
$\begin{array}{c}\text { (c) } 0.2 \mathrm{~mm} / \mathrm{s} \text { downward } \\
\text { flow }\end{array}$ & 0.000025 & 0.00025 & -0.31 & 0.62 \\
$\begin{array}{c}\text { (d) } 0.4 \mathrm{~mm} / \mathrm{s} \text { downward } \\
\text { flow }\end{array}$ & 0.0000087 & 0.000084 & -0.63 & 1.31 \\
\hline
\end{tabular}

In Fig. 3, we can first compare the results from the top to the bottom to observe the effect of the opposite flow directions, where Figs. 3(a) and 3(b) show the results for the upward flow while Figs. 3(c) and 3(d) show the results for the downward flow. As expected, in the two top figures, the Doppler power spectral profiles are both shifted correspondingly to the right side of $5 \mathrm{~Hz}$ or $15 \mathrm{~Hz}$, and in the two bottom figures, the profiles are both correspondingly shifted to the left side of $5 \mathrm{~Hz}$ or $15 \mathrm{~Hz}$. As can be further figured out from Table2, the fitted Doppler shifts $\delta$ correctly reflect not only the flow direction but also the relation of the doubled flow speed. Moreover, the fitted width $\sigma$ is about two times of the corresponding $\delta$, and is larger than those obtained for the horizontal flow (see Table 1). If we use the formula $(0.2 \mathrm{~mm} / \mathrm{s}) \times 1.9 \mathrm{MHz} /(1.5 \mathrm{~mm} / \mu \mathrm{s})$ to estimate $\delta$ of the $0.2 \mathrm{~mm} / \mathrm{s}$ flow (where $1.9 \mathrm{MHz}$ is the modulation frequency and $1.5 \mathrm{~mm} / \mu \mathrm{s}$ is the sound speed in water), the result is about $0.25 \mathrm{~Hz}$ which is close to the extracted values of $0.35 \mathrm{~Hz}$ and $0.31 \mathrm{~Hz}$ shown in Table2. We note here that this about 20\% discrepancy for the flow velocity could be mainly induced by the uneven distribution of the flowing beads across the cross section of the flow tube: we observe the beads are more likely flocking near the center of the tube.

As for the available spectral information, we can see from Table 2 that the ratio of $a_{2} / a_{1}$ for the $0.2 \mathrm{~mm} / \mathrm{s}$ upward flow is about 14.6 which is very close to the values obtained in the Horizontal flow (shown in Table 1). For the other three flow measurements, the ratios are all close to 10.0. We think the discrepancy is mainly due to more than 7 times decreasing of the signal to noise ratio for the vertical flow cases compared with the horizontal flow cases. Obviously, the low signal to noise ratio affects the accuracy more for extracting $a_{1}$ which is related to the photoacoustic Doppler signal excited by the blue diode laser.

\section{Discussion and conclusions}

Comparing the photoacoustic Doppler power spectra shown in Fig. 3 to those in Fig. 2 as well as the accompanying Gaussian fitting results listed in Table 2 to those in Table 1, we find that overall the photoacousitic signal measured from the vertical portion of the flow tube is more than 7 times lower than that from the horizontal portion. This is reasonable since the cylinder shaped flow portion illuminated by the laser spot generates the photoacousitc wave mainly propagating along the direction perpendicular to the flow tube. To get the sufficient signal to noise ratio for the vertical flow measurement, especially for the red dyed bead sample, the modulated laser intensity needs to be increased at least 10 times. This can be achieved by focusing the laser spot onto a short line instead of the circular spot when the laser diode power is still kept at the same level.

For our current study, 3 runs to 5 runs have been repeated for each flow measurement setting, and similar results have been obtained. As demonstrated, both vertical flow measurement and horizontal flow measurement can be used to analyze the flow speed. For the former, both of the peak Doppler shift and the Doppler broadening width reflect the flow speed well, and for the latter the Doppler broadening width alone accurately reflects the flow speed. As for the mechanism of forming the Doppler broadening, 
although the Doppler angle distribution due to the focused ultrasound transducer and the velocity distribution of the flowing beads both contribute, the broadening width of the horizontal flow is mainly caused by the Doppler angle distribution while the broadening width of the vertical flow is mainly determined by the flow velocity distribution. Since our major purpose here is to demonstrate the capability of analyzing the spectral information by applying dual wavelengths, we have not yet examined more flow speed settings in order to establish a more accurate analysis model for directly extracting the profile of the flow velocity distribution.

A further experiment under considering is to measure the mixture of the dyed beads with two different colors. If the beads with different colors own different velocity distributions, it can be anticipated that the two Doppler power spectra located at the demodulated frequencies of the blue laser diode and red laser diode will show different shapes. By the unmixture analysis, it is then possible to extract the different flow velocity distributions of the different color beads. This type of measurement will show more strongly the advantage of the multi-wavelength Doppler flow measurement than the single wavelength measurement.

In summary, our experimental results have shown unambiguously that by modulating two laser diodes with different wavelengths at two different modulation frequencies, the Doppler power spectra containing the absorption spectroscopic information of the flow sample can be obtained. By analyzing the Doppler power spectra with the simplified Gaussian fitting formulas, the color of the flow dyed beads can be easily differentiated. This study paves the way for further establishment of the multi-wavelength experiment system to get more complete absorption spectral information. Applying this spectroscopic technique in the blood flow measurement to fulfill the goal of accurately measuring the flow speed and at the same time, differentiating the oxygenation of the blood cells [19] can be anticipated by incorporating the tight focusing of the laser excitation and the high sensitive ultrasound detection [20].

\section{Acknowledgment}

This work is supported by the National Natural Science Foundation of China (Grant No. 11774256) and the Natural Science Foundation of Guangdong Province (Grant No. 2018B03031104).

Open Access This article is distributed under the terms of the Creative Commons Attribution 4.0 International License (http://creativecommons.org/licenses/by/4.0/), which permits unrestricted use, distribution, and reproduction in any medium, provided you give appropriate credit to the original author(s) and the source, provide a link to the Creative Commons license, and indicate if changes were made.

\section{References}

[1] L. V. Wang and L. Gao, "Photoacoutic microscopy and computed tomography: from bench to bedside," Annual Review of Biomedical Engineering, 2014, 16: 155-185.

[2] J. Weber, P. C. Beard, and S. E. Bohndiek, "Constrast agents for molecular photoacoustic imaging," Nature Method, 2016, 13(8): 639-650.

[3] A. B. E. Attia, G. B. alasundaram, M. Moothanchery, U. S. Dinish, R. Z. Bi, V. Ntziachristos, et al., "A review of clinical photoacoustic imaging: current and future trends," Photoacoustics, 2019, 16: 100144.

[4] K. Maslov and L. V. Wang, "Photoacoustic imaging of biological tissue with intensity-modulated continuous-wave laser," Journal of Biomedical Optics, 2008, 13(2): 024006.

[5] G. Langer, B. Buchegger, J. Jacak, T. A. Klar, and T. Berer, "Frequency domain photoacoustic and fluorescence microscopy," Biomedical Optics Express, 2016, 7(7): 2692-2702.

[6] P. LeBoulluec, H. L. Liu, and B. H. Yuan, "A cost-efficient frequency-domain photoacoustic imaging system," American Journal of Physics, 2013, 81(9): 712-717.

[7] O. Hugon, B. V. D. Sanden, M. Inglebert, O. Jacquin, C. Misbath, and E. Lacot, "Multi-wavelength photoacoustic microscopy in the frequency domain for simultaneous excitation and detection of dyes," Biomedical Optics Express, 2019, 10(2): 932-943.

[8] P. Mohajerani, S. Kellnberger, and V. Ntziachristos, 
"Frequency domain optoacoustic tomography using amplitude and phase," Photoacoustic, 2014, 2(3): 111-118.

[9] S. Kellnberger, D. Soliman, G. J. Tserevelakis, M. Seeger, H. Yang, A. Karlas, et al., "Optoacoustic microscopy at multiple discrete frequencies," Light: Science \& Applications, 2018, 7(1): 1-12.

[10] S. Y. Liu, K. Tang, X. H. Feng, H. R. Jin, F. Gao, and Y. J. Zheng, "Toward wearable healthcare: a miniaturized 3D imager with coherent frequencydomain photoacoustics," IEEE Transactions on Biomedical Circuits and Systems, 2019, 13(6): 1417-1424.

[11] S. S. S. Choi and A. Mandelis, "Review of the state of the art in cardiovascular endoscopy imaging of atherosclerosis using photoacoustic techniques with pulsed and continuous-wave optical excitations," Journal of Biomedical Optics, 2019, 24(8): 080902.

[12] H. Fang, K. Maslov, and L. V. Wang, "Photoacoustic Doppler effect from flowing small light-absorbing particles," Physical Review Letters, 2007, 99(18): 184501.

[13] H. Fang, K. Maslov, and L. V. Wang, "Photoacoustic Doppler flow measurement in optically scattering media," Applied Physics Letters, 2007, 91(26): 264103.

[14] H. Fang and L. V. Wang, "Photoaoustic Doppler effect and flow sensing. Photoacoustic Imaging and Spectroscopy," L. V. Wang, Ed. Boca Raton, FL: CRC Press, 2009: 19-24.

[15] A. Sheinfeld, S. Gilead, and A. Eyal, "Photoacoustic Doppler measurement of flow using tone bust excitation," Optics Express, 2010, 18(5): 4212-4221.

[16] A. Sheinfeld, S. Gilead, and A. Eyal, "Simultaneous spatial and spectral mapping of flow using photoacoustic Doppler measurement," Journal of Biomedical Optics, 2010, 15(6): 066010.

[17] Y. Tong, H. C. Zhao, H. Fang, Y. Q. Zhao, and X. C. Yuan, "Flow angle dependent photoacoustic Doppler power spectra under intensity-modulated continuous wave laser excitation," AIP Advances, 2016, 6(2): 025109.

[18] P. J. Van den Berg, K. Daoudi, and W. Steenbergen, "Review of photoacoustic flow imaging: its current state and its promises," Photoacoustics, 2015, 3(3): 89-99.

[19] L. D. Wang, K. Maslov, and L. V. Wang, "Single-cell label-free photoacoustic flowoxigraphy in vivo," Proceedings of the National Academy of Sciences, 2013, 110(15): 5759-5764.

[20] F. Yang, W. Song, C. L. Zhang, C. J. Min, H. Fang, L. P. Du, et al., "Broadband graphene-based photoacousitc microscopy with high sensitivity," Nanoscale, 2018, 10(18): 8606-8614. 